\title{
Design and development of open source portable multi-functional three-dimensional bio-printing extrusion system for hydrogel-based scaffolds fabrication
}

\section{Pouya Behrouzi}

Bushehr University of Medical Sciences

Mahmood Zali

Bushehr University of Medical Sciences

Mahdi Muhaddesi

Bushehr University of Medical Sciences https://orcid.org/0000-0001-5408-3954

Alireza Hashemi

Bushehr University of Medical Sciences

Arezoo Khoradmehr

Bushehr University of Medical Sciences

Amin Torabi Jahromi

Persian Gulf University

Iraj Nabipour

Bushehr University of Medical Sciences

Amin Tamadon ( $\square$ amintamaddon@yahoo.com )

Bushehr University of Medical Sciences https://orcid.org/0000-0002-0222-3035

\section{Article}

Keywords: 3D Bio-printing, Biomedical engineering, Tissue engineering, Organ 3D Printing

Posted Date: March 1st, 2021

DOl: https://doi.org/10.21203/rs.3.rs-181593/v1

License: (c) (i) This work is licensed under a Creative Commons Attribution 4.0 International License. Read Full License 


\section{Abstract}

Three-dimensional (3D) bio-printing has been shown up as a state of the art and creative technological solution to nowadays tissue engineering and stem cell research challenges toward human tissue regeneration and construction of artificial living organs. Thereby, using hydrogel-based bio-inks to 3D print living microenvironments is a crucial strategy to reconstruct basically functional living scaffolds in order to shape human living organs based on digital 3D computer-aided design (CAD) inputs. The focus of this paper lies on design and development of a portable multi-functional 3D bio-printing extruder for fabrication of hydrogel-based highly complex living tissues using advanced methods invented along this study. The presented article, precisely optimizes the process of fabricating 3D printed scaffolds by redesigning of an integrated gel-extrusion system capable of controlling the adjustability of thermal condition of bio-inks. Also a UV crosslink module is utilized in the bottom of the extruder to cure hydrogel scaffolds consisting of photo-reacting agents to provide a novel bio-printing experience for end users. As a result, the integrated extrusion system is easily portable and compatible with almost any computer numerical control (CNC) machine. Therefore, it could be simply installed on or removed from any CNC machine or fused deposition modeling (FDM) 3D printing system considering that all the control units remain adjustable. The whole system parameters and the performance of tissue fabrication regarding this developed portable multi-functional 3D bio-printing extruder have been tested and practically confirmed. The thermal control system performance has also been simulated using finite element analysis (FEA) and computational fluid dynamics (CFD) methods. Thus, certified documents have been provided and depicted in this paper.

\section{Introduction}

According to the recent advances in stem cell biology and biotechnology and its convergence with tissue engineering and additive manufacturing (1), a significant amount of demands give rise to the formation of a combined theory of 3D bio-printing microenvironments for the aim of human living tissue replacements (2). The 3D bio-printing is the process of layer-by-layer fabrication of bio-compatible materials with or without cells in order to form a construct for biomedical applications (3). Artificially constructed microenvironments are being utilized in pharmaceutical drug screening platforms as a simulated counterpart of actual in-vivo tissues and organs $(4,5)$. In addition, they are playing a crucial role in tissue engineering and organ replacement as a clinical usage of these bio-fabricated functional tissues (6).

In fact, hydrogel-based bio-inks are the most common substrates that have been used in 3D bio-printing of functional living tissues (7). Each of these bio-inks are made up of gel-based materials and the composition of biological agents which have their specific physio-chemical characteristics due to its synthesizing method and building blocks (8). Considering that every bio-ink has its own values of 3D bioprinting parameters and specified level of viscosity, melting point, gelation point and overall printability (9), design and development of a portable multi-functional 3D bio-printing extruder that has a high level of flexibility to become compatible with a variety of bio-inks and accordingly vast spectrum of 
applications is a step forward toward 3D printing of geometrically complex living organisms using extrusion-based bio-printing $(10,11)$.

The aim of this work presented in this paper is to design and develop of portable multi-functional 3D bioprinting extruder for constructing hydrogel-based complex living tissues for organ replacement and drug screening applications. Thus, the developed device has the ability to precisely control the thermal parameters of bio-ink that enables the user to 3D bio-print extremely porous microenvironments using the majority of bio-materials with different characteristics (12). By taking into account that cell laden hydrogels are the type of bio-inks that has many applications in bio-engineering of in-vivo organs (13), the stem cells are able to be loaded into syringe which is mounted on the novel multi-functional extruder because the system has the ability to maintain the thermal condition needed for the viability of cells that are loaded on (14).

In the process of 3D bio-printing some types of functional living bio-structures such as extracellular matrix (ECM) (15), osteoblastic scaffold with trabecular pattern which have a highly complex geometry (16), and maintaining the scaffolds to be fixed and cured layer-by-layer during the fabrication process are challenges in 3D bio-printing methods. According to the recent advancements in development of hydrogel-based bio-inks for tissue engineering and 3D bio-printing applications, photo initiators and UV absorbers are utilized to formulate a photo-reacting hydrogels (17). Gelatin and meta-acrylate based bioinks are the most common 3D bio-printing substrates for 3D printing of complex biological constructs for the aim of developing drug delivery platform and organ replacement (18). Considering the characteristics of these sort of bio-inks, they are curable by energy that is provided by UV light spectrum to the constructs which are made up of these gel-based materials. Thus, a UV light crosslinking embedded platform is attached at the bottom of the device where is equipped with a $395 \mathrm{~nm}$ wavelength power LED lights to do the crosslinking of the hydrogel consisting of photo-reacting agents during the 3D fabrication process.

In some cases, the complexity of a living tissue such as vessels existing in the middle of the scaffold or the multi-material 3D bio-printing of a functional 3D bio-fabricated organ causes serious limitations in the 3D bio-printing of such complex microenvironments due to the drawbacks of existing commercial tools and available techniques in 3D bio-printers (19). Also, existing open source developed 3D bio-printing systems have some disadvantages regarding their thermal control mechanism and offer wide range of inflexibilities when it comes to more complex applications (20). This paper eliminates some of the limitations of the existing tools and methods by providing an ultra-precise thermal controlling unit equipped by an integrated heating and cooling system embedded on the developed portable multifunctional 3D bio-printing extruder that is able to maintain the temperature stable in the interval of $0-100^{\circ}$ C. Therefore, the system is adoptable to a vast spectrum of bio-materials with a different gelation points and characteristics.

\section{Materials And Methods}

\section{Overall design and implementation}


Building blocks of the portable multi-functional 3D bio-printing extruder were all made out of aluminum metal sheets and bulk aluminum which was manufactured by utilizing CNC milling machine. All the metal parts which form the body of the device had been mounted on the main plate which made out of a $3 \mathrm{~mm}$ aluminum metal sheet manufactured by an advanced milling machine (Fig. 1A). Aluminum cubic holder which was mounted on the base plate of the extruder was the most important building block of the mechanical hardware (Fig. 1B). It has a direct contact with syringe, cylindrical $30 \mathrm{~W} 12 \mathrm{~V}$ heating element, NTC $100 \mathrm{k} \Omega$ thermistor, and $4 \times 4 \mathrm{~cm}$ water block. The water block was linked to an external thermoselectric liquid cooling system to cool down the cubic holder (Fig. 1C). A glass syringe was considered to be used due to their compatibility with variety of hydrogels with different gelation points (Fig. 1D). Therefore, the syringe was not going to be melted or degraded due to heating condition needed for the printability of bio-inks with specific thermal setting. A cylindrical slot was provided on the right side of the aluminum cubic holder for the installation of heating element (Fig. 1E). On the other hand, a deep tiny slot was also provided on the aluminum cubic holder very close to the syringe output to control the temperature (Fig. 1F). The mentioned cubic holder had a direct contact with the $4 \times 4 \mathrm{~cm}$ water block to cool the syringe for applications in reverse gelation system for vessel printing by a thermal conductive silicon pad installed between them (Fig. 1G). Furthermore, a UV-LED curing system was provided on the bottom aluminum plate of the portable multi-functional extruder that effected the crosslinking process during the printing time (Fig. $1 \mathrm{H}$ ). The aluminum cubic holder was thermodynamically isolated from the other parts by a heating insulation cotton pad to prevent energy loss and minimum the thermal control system tolerance (Fig. 1I) (21). Finally, the whole system was covered by a $1 \mathrm{~mm}$ aluminum shield to protect the system from external destructions such as humidity and to enhance the stability of thermal control system (Fig. 1J).

\section{Syringe thermal control system}

The thermal control system in the portable multi-functional 3D bio-printing extruder includes two major parts, namely heating and cooling systems. They were all embedded on a pack of electro-

thermomechanical system that is equipped with a $100 \mathrm{k} \Omega$ thermistor as a sensor. It provides the system with thermal control data that enables the 3D bio-printer to control and maintain the thermal setting needed for a specific hydrogel which loaded into the syringe part.

The cylindrical heating element was encapsulated in the aluminum cubic holder slot which was fixed on the right side of the cube to properly transfer the heat to the glass syringe due to the extremely high heat conductivity of the 6061 aluminum alloy which was applied to manufacture the cubic holder. The cylindrical heating element have to precisely fit the slot provided on the right hand side of the cube to flawlessly transfer the heat and minimize the energy loss in the system. To do so, a heat conductive silicon pad with $6 \mathrm{~W} /(\mathrm{m} \cdot \mathrm{K})$ is applied in this research to bound the surface of the heater and the aluminum cubic holder. Therefore, the heater must be shielded by the pad and then plugged into the slot to get bonded (Fig. 2A).

A $4 \times 4 \mathrm{~cm}$ water block was installed on the back of the aluminum cubic holder. The water block was linked to an external Peltier-Thermoelectric Liquid cooler (LC-200 TE Technology, INC.) by a silicon tube 
and a water pump that moves the liquid through the loop with $1.6 \mathrm{~L} / \mathrm{min}$ flow rate. An antifreeze based water liquid flow is run into the close loop flow system to transfer the energy from the aluminum cubic holder to the external thermoelectric liquid cooler (Fig. 2B). Antifreeze liquid material is also utilized to prevent the freezing of the liquid in the silicon tube through its flow loop across thermoelectric liquid cooler module which is able to remove heat with approximately $100 \mathrm{~W}$ power with ambient temperature of $25^{\circ} \mathrm{C}$. This structure has been utilized to cool down the aluminum cubic holder in order to lower the temperature of syringe and the material loaded into it (Fig. 2B). As a result, the extruder was able to cool down the hydrogel to enhance the viscosity of the bio-ink which has a low viscosity and printability in the room temperature. Finally, the inner surface of glass syringe slot which was provided on the aluminum cubic holder is covered by the same silicon pad that was used for the bonding of cylindrical heating element and cubic holder surface to properly transfer the energy between syringe and the aluminum cubic holder. Therefore, the corresponding temperature is continuously measured by the NTC $100 \mathrm{k} \Omega$ resistant thermistor and signals are transferred to the micro-processing unit (ATmega2560). It controlled and maintained the thermal condition set as a target for a specific application.

\section{UV light crosslinking module}

A UV crosslinking module is embedded in the portable multi-functional 3D bio-printing extruder system. It utilizes a 1-3 W UV power LED with $395 \mathrm{~nm}$ wavelength to automatically cure the 3D bio-printed scaffold in order to higher the precision and fidelity of the 3D biological constructs (22). The UV light curing module is controlled by the micro-processing unit (ATmega2560). The users are able to customize the UV crosslinking procedure by modification of G-Code communication lines while rendering the standard triangle language (STL) file using 3D slicer software. The UV crosslinking module is located at the bottom of the multi-functional extruder and is fixed on the bottom plate. The 1-3 W UV power LED is used in the module which transmits $395 \mathrm{~nm}$ UV spectrum to do the curing procedure. The UV LED 120-degree lens is installed to perfectly cure the hydrogel-based constructs with a homogeneous light transmission.

\section{System wiring}

Totally, the developed portable multi-functional 3D bio-printing extrusion system had three major parts. Each part consists of sub-systems with a group of components which were linked together toward a specific purpose. Thermal control system, movement system, and UV crosslinking module were the three major subsystems that were embedded on an integrated 3D bio-printing system. Thermal control system includes a $30 \mathrm{~W} 12 \mathrm{~V}$ cylindrical heating element, and NTC thermistor to continuously control the parameters. NEMA-17 bipolar stepper motor wires which were connected to the pins 1-4 on P1 4-position connector (Fig. 3A). Considering the power consumption of the $12 \mathrm{~V} 30 \mathrm{~W}$ cylindrical heating element, a more tolerable port and wiring is needed. Thus, we utilized a 2-position terminal block to wire this key element in the heating system. Cylindrical heating element wires were soldered to pin 1 and 2 on PADS1 inputs (Fig. 3B). UV crosslinking module made up of a $33 \Omega$ resistor and a 1-3 W $395 \mathrm{~nm}$ UV power LED. The UV module connected to the P5 connector which output the wiring to the pins $3-4$ of P3 connector. NTC $100 \mathrm{k} \Omega$ Thermistor connected to the P4 connector which output the wiring to the pins $1-2$ of P3 connector (Fig. 3C). 


\section{Mechanical movement system}

The mechanical motion control of the system was carried base on the generated G-Code uploaded on the micro-processing unit. The whole extrusion system was mounted on the $\mathrm{X}$-axis of the fused deposition modeling (FDM) 3D bio-printing platform. Axial movement of the system is a combination of motions carried by the $X, Y$ and $Z$ axes which enables the bio-ink to be deposited on the bed plate in threedimensional movements (Fig. 4A). The deposition of hydrogel-based bio-ink is performed by the mechanical pressure generates by the bipolar Nema- 17 stepper motor and transmits by the $8 \times 5 \mathrm{~mm}^{2}$ aluminum coupling system to the syringe slider (Figs. 4B and $4 \mathrm{C}$ ). The generated pressure makes the slider to move upward and downward to do the injection and deposition of hydrogel on the bed plate (Fig. 4D). The extrusion rate of the system was adjustable by modification of the bipolar stepper motor calibration in the 3D printing frameware such as open-source Marlin and GRBL. Details of the mechanical components of the system is listed on the Supplementary Table S1.

\section{Portability}

All the system wiring implemented on the multi-functional 3D bio-printing extrusion system for constructing hydrogel-based complex living organs are portable and easy to install on a variety of CNC machines and 3D printing platforms with different control boards. The system has three main ports including J1 2-pin terminal block, P2 and P3 4-pin connector which their outputs are connected to the responsible GPIO on corresponding control board (Fig. 5A). Pins 1-4 of P2 connector are considered for the bipolar stepper motor pinouts which was wired to the control board GPIO responsible for extruder motor controlling the flow rate and deposition speed (Fig. 5B). Furthermore, pins 1-2 on P3 connector considered for the NTC $100 \mathrm{k} \Omega$ Thermistor pinouts that were connected to the responsible GPIO on the corresponding control board to control the thermal condition of the aluminum cubic holder. Pins 3-4 on P3 connector were considered for the UV LED crosslinking module which is connected to an auxiliary pin on the corresponding control board by putting a metal-oxide-semiconductor field-effect transistor (MOSFET) between pin 3 and the auxiliary pin on the control board (Fig. 5C). Thereby, its light density and running time were controllable through G-Code communication port responsible for that corresponding auxiliary pin. Finally, J1 terminal block 2-pin connector is utilized to run the high consuming $30 \mathrm{~W} 12 \mathrm{~V}$ Heating Element (Fig. 5D). Details of the electronic components of the system is listed on the Supplementary Table S2.

\section{Results And Discussions}

As a result, the performance of the system in each sub-system has been tested and the results confirm that the developed portable multi-functional 3D bio-printing extruder is able to perfectly 3D bio-fabricate functional living microenvironments for drug screening and tissue engineering applications. After all, the whole integrated system including the subsystems such as mechanical movement system, UV crosslinking module, thermal condition control system and the portability of the developed extruder and its compatibility to CNC machines and 3D FDM Printer has been tested. Furthermore, syringe heating 
system has been simulated using SOLIDWORKS Simulation built-in plugin based on the Finite Element Analysis (FEA) (23). All the surfaces are assumed to be in contact with air with room temperature and heat transfer coefficient of $25 \mathrm{~W} / \mathrm{m} 2 \mathrm{~K}$ which is a normal convection rate in room temperature (24). The performance of the system has been measured and confirmed that it was able to heat the material environment up to $37^{\circ} \mathrm{C}$ using $30 \mathrm{~W} 12 \mathrm{~V}$ heating element and the heat has been perfectly transferred to the aluminum cubic holder and the glass syringe with a maximum tolerance of $\pm 2^{\circ} \mathrm{C}$ referring to the system simulation plots with respect to corresponding parameters (Figs. 6A and 6B). The system is able to reach higher temperatures near $100^{\circ} \mathrm{C}$ as it is depicted in the plots in Supplementary Figures S1A and S1B. In addition, the syringe cooling system had been simulated using SOLIDWORKS Flow Simulation plugin based on the Computational Fluid Dynamics (CFD) method (25). The liquid cooling system has the ability to cool down the bio-ink environment down to $0^{\circ} \mathrm{C}$ in the 78th second of the simulation running time (Supplementary Video S1). The stability of the temperature with a maximum tolerance of \pm $2^{\circ} \mathrm{C}$ has been proved referring the system simulation plots with corresponding parameters (Figs. $6 \mathrm{C}$ and $6 \mathrm{D})$. Mechanical motion of the extrusion system which is made up of a bipolar stepper motor, $8 \mathrm{~mm}$ lead screw and the $8 \times 5 \mathrm{~mm}$ coupling has been tested and confirmed that the deposition of the bio-inks loaded into the glass syringe has a smooth and accurate performance to 3D bio-fabricate living constructs with complex structures. The presented method has a significant advantage in comparison to the air pressure based deposition model due to its high resolution and super precise deposition and flexibility of the flow rate configuration in a vast spectrum of hydrogels with different viscosity and mechanical properties. The UV crosslinking module accurately worked during the printing time of the hydrogel-based scaffolds and its performance of UV light transmission measured by a UV sensitive material based on gelatin methacrylate. The photo sensitive bio-ink is 3D printed with $200 \mathrm{~mm} / \mathrm{min} X Y$ plotting speed and then cured by the $395 \mathrm{~nm}$ UV light spectrum within 120 seconds of exposure time in the room temperature. The 3D bio-printed hydrogel-based construct is observed under the loop microscope and the fidelity of the structure had been confirmed (Fig. 7).

\section{Conclusions}

In conclusion, the fabrication of 3D functional biological scaffolds could enable the mankind to cover a vast spectrum of challenges in tissue engineering and organ printing applications as well as drug screening and organ replacements. To do so, an embedded portable multi-functional 3D bio-printing platform is designed and developed that its multi-functionality and portability has been tested and its performance has been confirmed due to the data release as result of this study. Thereby, the end-user of the developed system is going to have the ability to apply this device to 3D bio-print high fidelity, biocompatible and cell viable microenvironment to reach a perfectly optimized parameters of a 3D biological construct that can be utilized for the specific applications mentioned before in this paper.

\section{Declarations}

\section{Supplementary DATA}


Supplementary Tables S1 and S2

Supplementary Figures S1A and S1B

Supplementary Video S1

\section{Acknowledgments}

Not applicable.

\section{Declarations}

Author Pouya Behrouzi, Mahmood Zali, Mahdi Muhaddesi, and Alireza Hashemi were employed by the company PerciaVista Co. The remaining authors declare that the research was conducted in the absence of any commercial or financial relationships that could be construed as a potential conflict of interest.

\section{Consent for publication}

Not applicable.

\section{Availability of data and material}

All the users have open access to the available data and material which includes, 2D industrial drawing files of mechanical parts, 3D files of 3D printing parts, and electronic PCB schemes of the system. Link: https://doi.org/10.7910/DVN/OIIOEQ

\section{Funding}

This study was funded by a grant from The Council for Development of photonic, laser, advanced materials and manufacturing technologies, affiliated to the Iranian Vice Presidency for Science and Technology.

\section{Competing interests}

The authors declare no conflicts of interest.

\section{Authors' contributions}

1. B., M. Z., M. M., A. H., A. T., and I. N. conceived and designed the format of the manuscript. M. Z., M. M, A. H., A. K., and A. T. J. analyzed the data, drafted and edited the manuscript. P. B., M. Z., M. M., and $A$. H. produced the figures, tables and materials of this paper. All the authors reviewed the 
manuscript and all of them contributed to the critical reading and discussion of the manuscript. All authors have read and agreed to the published version of the manuscript.

\section{Acknowledgments}

Not applicable.

\section{References}

1. Mota C, Puppi D, Chiellini F, Chiellini E. Additive manufacturing techniques for the production of tissue engineering constructs. Journal of tissue engineering and regenerative medicine. 2015;9(3):174-90.

2. Machino R, Matsumoto K, Taniguchi D, Tsuchiya T, Takeoka Y, Taura Y, et al. Replacement of Rat Tracheas by Layered, Trachea-Like, Scaffold-Free Structures of Human Cells Using a Bio-3D Printing System. Advanced healthcare materials. 2019;8(7):1800983.

3. Chhaya MP, Poh PS, Balmayor ER, van Griensven M, Schantz J-T, Hutmacher DW. Additive manufacturing in biomedical sciences and the need for definitions and norms. Expert review of medical devices. 2015;12(5):537-43.

4. Peng W, Datta P, Ayan B, Ozbolat V, Sosnoski D, Ozbolat IT. 3D bioprinting for drug discovery and development in pharmaceutics. Acta biomaterialia. 2017;57:26-46.

5. Mazzocchi A, Soker S, Skardal A. 3D bioprinting for high-throughput screening: Drug screening, disease modeling, and precision medicine applications. Applied Physics Reviews. 2019;6(1):011302.

6. Nagarajan N, Dupret-Bories A, Karabulut E, Zorlutuna P, Vrana NE. Enabling personalized implant and controllable biosystem development through 3D printing. Biotechnology advances. 2018;36(2):52133.

7. Unagolla JM, Jayasuriya AC. Hydrogel-based 3D bioprinting: A comprehensive review on cell-laden hydrogels, bioink formulations, and future perspectives. Applied Materials Today. 2020;18:100479.

8. Park J, Lee SJ, Chung S, Lee JH, Kim WD, Lee JY, et al. Cell-laden 3D bioprinting hydrogel matrix depending on different compositions for soft tissue engineering: characterization and evaluation. Materials Science and Engineering: C. 2017;71:678-84.

9. Kyle S, Jessop ZM, Al-Sabah A, Whitaker IS. 'Printability'of Candidate Biomaterials for Extrusion Based 3D Printing: State-of-the-Art. Advanced healthcare materials. 2017;6(16):1700264.

10. Liu F, Liu C, Chen Q, Ao Q, Tian X, Fan J, et al. Progress in organ 3D bioprinting. International Journal of Bioprinting. 2018;4(1).

11. Ozbolat IT, Hospodiuk M. Current advances and future perspectives in extrusion-based bioprinting. Biomaterials. 2016;76:321-43.

12. Podshivalov L, Gomes CM, Zocca A, Guenster J, Bar-Yoseph P, Fischer A. Design, analysis and additive manufacturing of porous structures for biocompatible micro-scale scaffolds. Procedia Cirp. 
2013;5:247-52.

13. Hong N, Yang GH, Lee J, Kim G. 3D bioprinting and its in vivo applications. Journal of Biomedical Materials Research Part B: Applied Biomaterials. 2018;106(1):444-59.

14. Ouyang L, Yao R, Zhao Y, Sun W. Effect of bioink properties on printability and cell viability for 3D bioplotting of embryonic stem cells. Biofabrication. 2016;8(3):035020.

15. Ma X, Yu C, Wang P, Xu W, Wan X, Lai CSE, et al. Rapid 3D bioprinting of decellularized extracellular matrix with regionally varied mechanical properties and biomimetic microarchitecture. Biomaterials. 2018;185:310-21.

16. Tellis B, Szivek J, Bliss C, Margolis D, Vaidyanathan R, Calvert P. Trabecular scaffolds created using micro CT guided fused deposition modeling. Materials Science and Engineering: C. 2008;28(1):171-8.

17. Qin X, Hu Q, Gao G, Guan S. Characterization of UV-curable poly (ethylene glycol) diacrylate based hydrogels. Chemical Research in Chinese Universities. 2015;31(6):1046-50.

18. Pepelanova I, Kruppa K, Scheper T, Lavrentieva A. Gelatin-Methacryloyl (GelMA) hydrogels with defined degree of functionalization as a versatile toolkit for 3D cell culture and extrusion bioprinting. Bioengineering. 2018;5(3):55.

19. Bishop ES, Mostafa S, Pakvasa M, Luu HH, Lee MJ, Wolf JM, et al. 3-D bioprinting technologies in tissue engineering and regenerative medicine: Current and future trends. Genes \& diseases. 2017:4(4):185-95.

20. Banović L, Vihar B. Development of an extruder for open source 3D bioprinting. Journal of Open Hardware. 2018;2(1).

21. Shalev I, Barker RL. Analysis of Heat Transfer Characteristits of Fabrics in an Open Flame Exposure. Textile Research Journal. 1983;53(8):475-82.

22. Pereira RF, Bártolo PJ. 3D bioprinting of photocrosslinkable hydrogel constructs. Journal of Applied Polymer Science. 2015;132(48).

23. Glodová I, Lipták T, Bocko J. Usage of finite element method for motion and thermal analysis of a specific object in SolidWorks environment. Procedia Engineering. 2014;96:131-5.

24. Khalifa A-JN. Natural convective heat transfer coefficient-a review: II. Surfaces in two-and threedimensional enclosures. Energy conversion and management. 2001;42(4):505-17.

25. Kurowski P. Thermal Analysis with SOLIDWORKS Simulation 2019 and Flow Simulation 2019: SDC Publications; 2019.

\section{Figures}




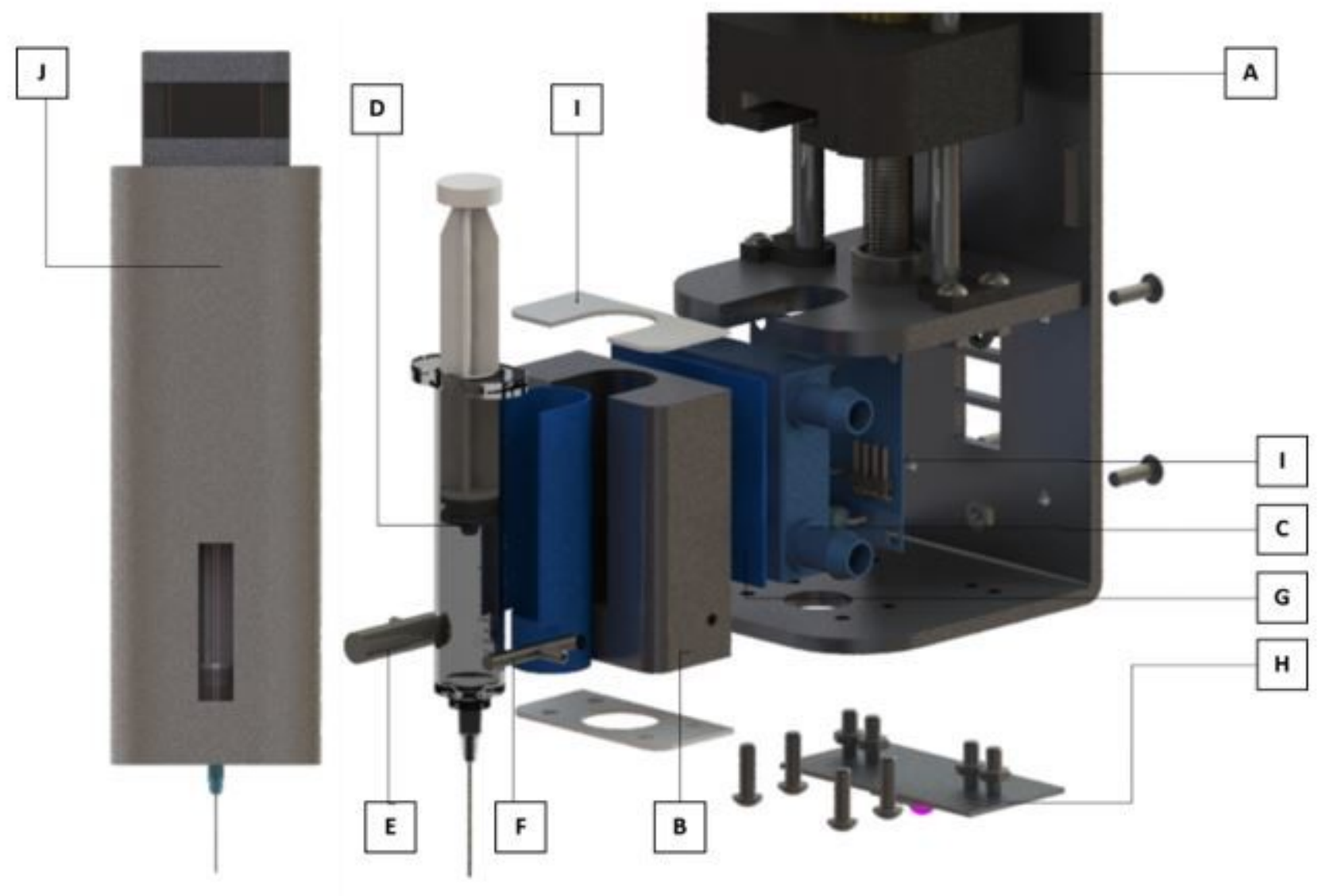

\section{Figure 1}

Overall design and development of the portable multi-functional 3D bio-printing extrusion system (A) Base plate. (B) Aluminum cubic holder. (C) $4 \times 4 \mathrm{~cm}$ water block. (D) Glass syringe. (E) $30 \mathrm{~W}$ cylindrical heating element. (F) NTC $100 \mathrm{k} \Omega$ thermistor. (G $4 \times 4 \mathrm{~cm}$ thermo-conductive silicon pad. (H) UV crosslinking module located in the bottom of the extruder mounted on the bottom plate. (I) Thermoresistance heating insulation cotton pad. (J) $1 \mathrm{~mm}$ aluminum shield.
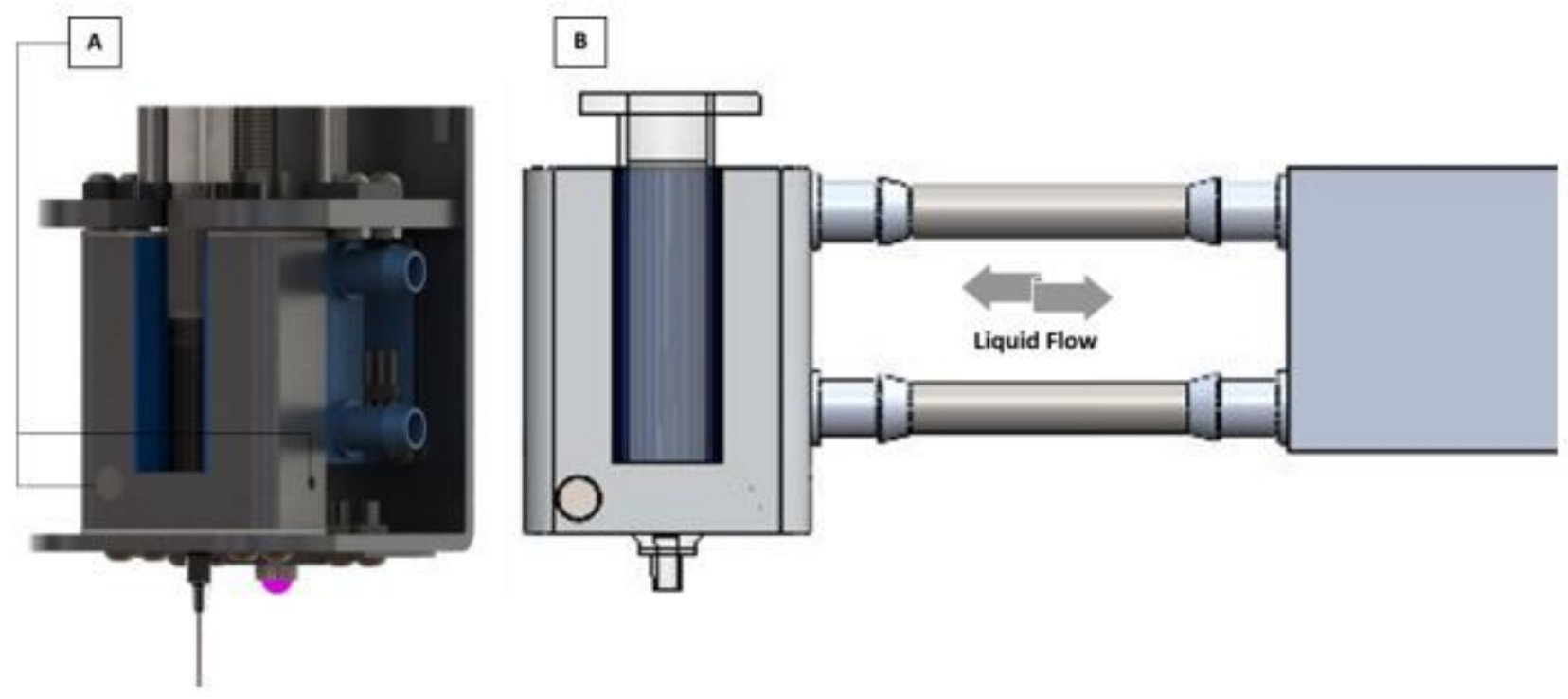
Figure 2

Thermal control system embedded on the device to heat and cool down the system in different applications. (A) Heating system includes a $12 \mathrm{~V}$ ceramic heater cartridge, and a NTC $100 \mathrm{k} \Omega$ thermistor. (B) Thermoelectric liquid cooler with close loop liquid flow to cool down the system.
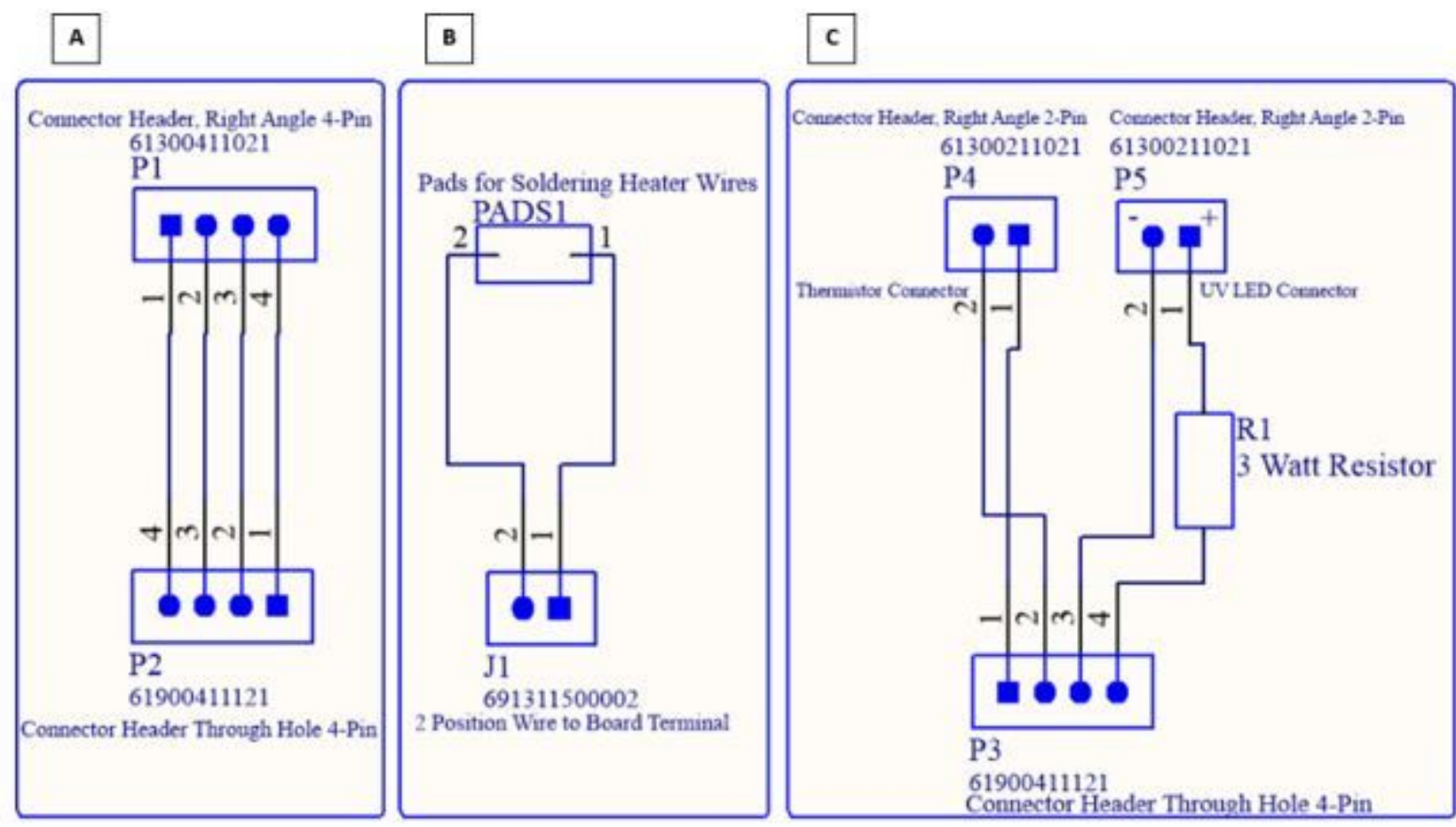

\section{Figure 3}

Integrated system wiring scheme including movement system, thermal control system, and UV crosslinking module. (A) Bipolar stepper motor connections. (B) $30 \mathrm{~W}$ cylindrical heater wiring to PADS1. (C). NTC thermistor and $395 \mathrm{~nm}$ UV LED wirings to P3 connector.

A

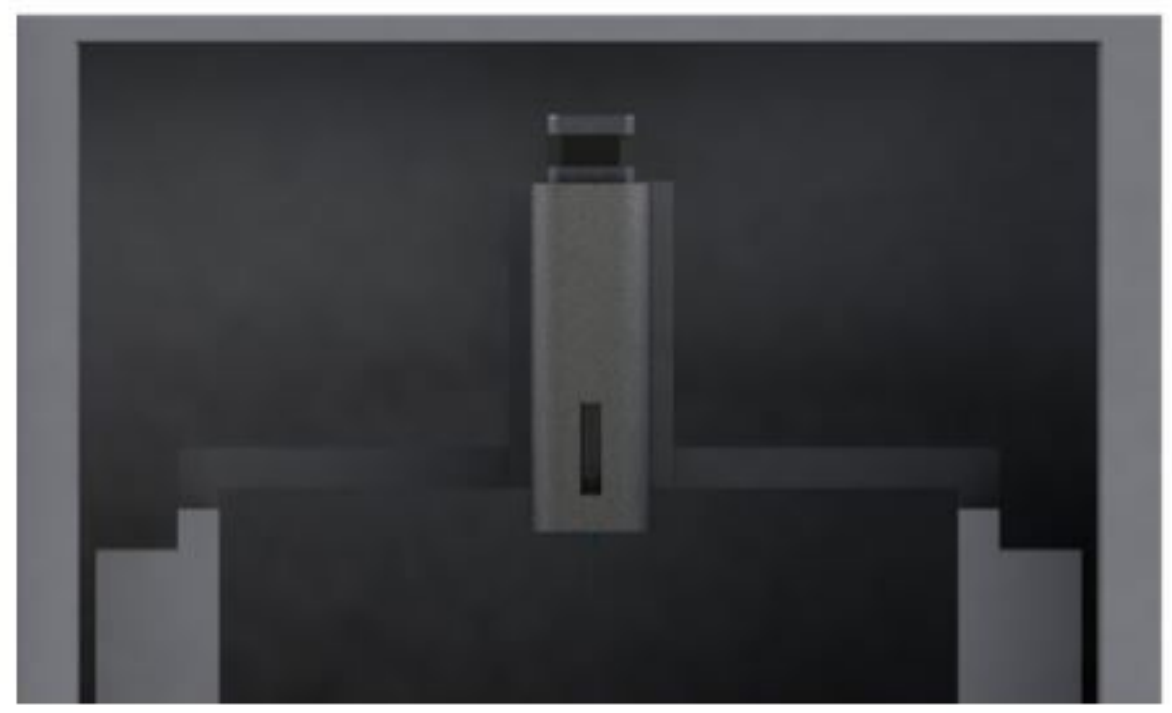

B

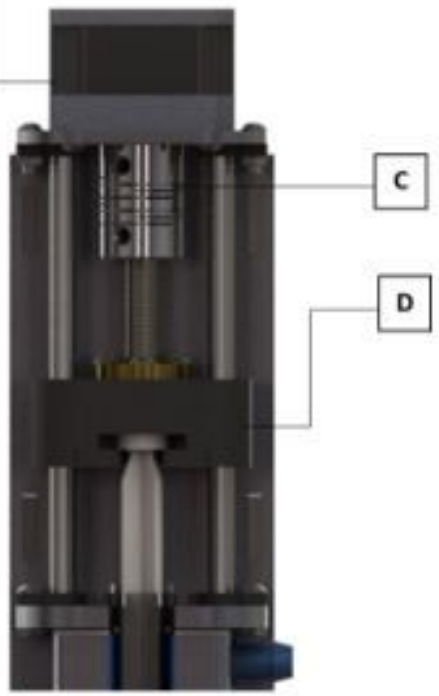




\section{Figure 4}

Mechanical movement system of multi-functional 3D bio-printing extrusion system. (A) Mounted portable multi-functional 3D bio-printing extrusion system for constructing hydrogel-based complex living organs on the $\mathrm{X}$ axis. (B) Nema-17 stepper motor that generates the mechanical pressure to do the extrusion. (C) $8 \times 5 \mathrm{~mm}$ coupling system that transmits the mechanical pressure generated by the bipolar stepper motor to the $8 \mathrm{~mm}$ lead screw for the aim of syringe slider movement. (D) Syringe slider that utilize the mechanical pressure generated by the bipolar stepper motor to move the slider downward or upward.

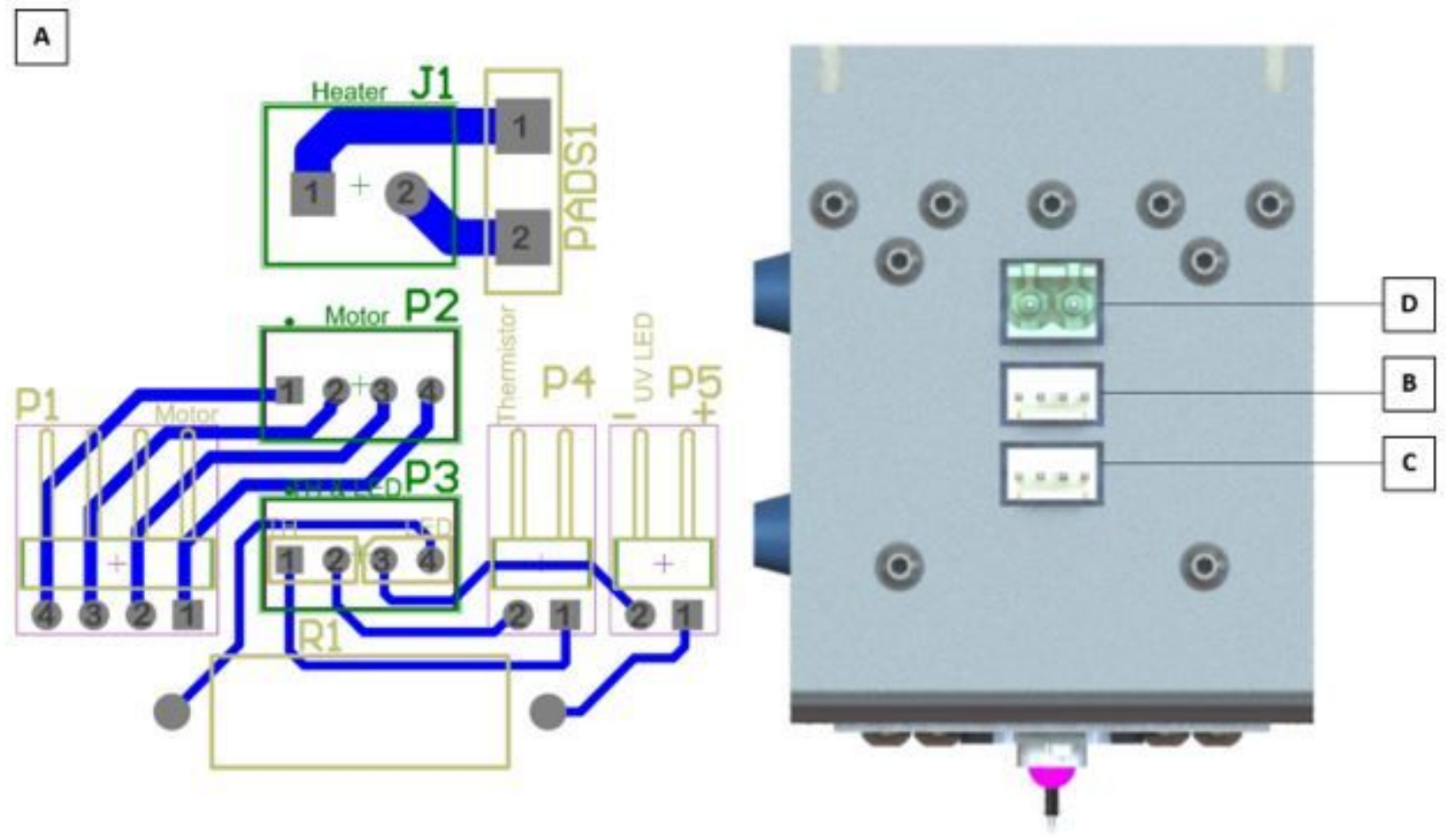

Figure 5

System portability scheme and Pinouts. (A) Overall PCB scheme of 3 major ports of the system. (B) P2, bipolar stepper motor port. (C) NTC $100 \mathrm{k} \Omega$ Thermistor and UV light crosslinking module integrated port. (D) PAD1, $30 \mathrm{~W} 12 \mathrm{~V}$ ceramic heating element port. 

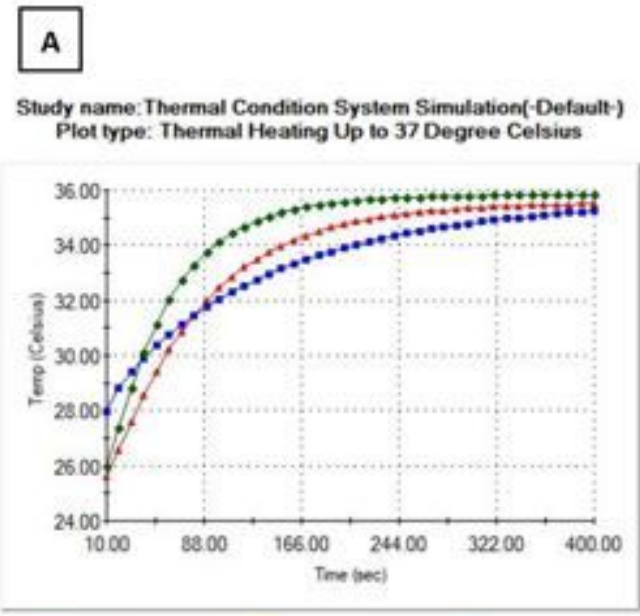

$\longrightarrow$ Node $18323 \longrightarrow$ Node $24312 \longrightarrow$ Node 11935

254708,362543
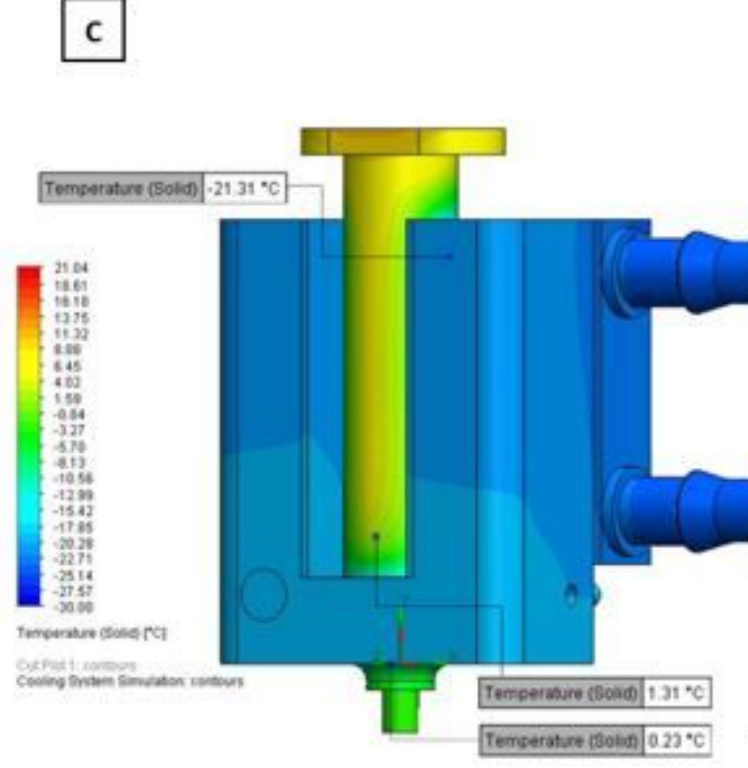
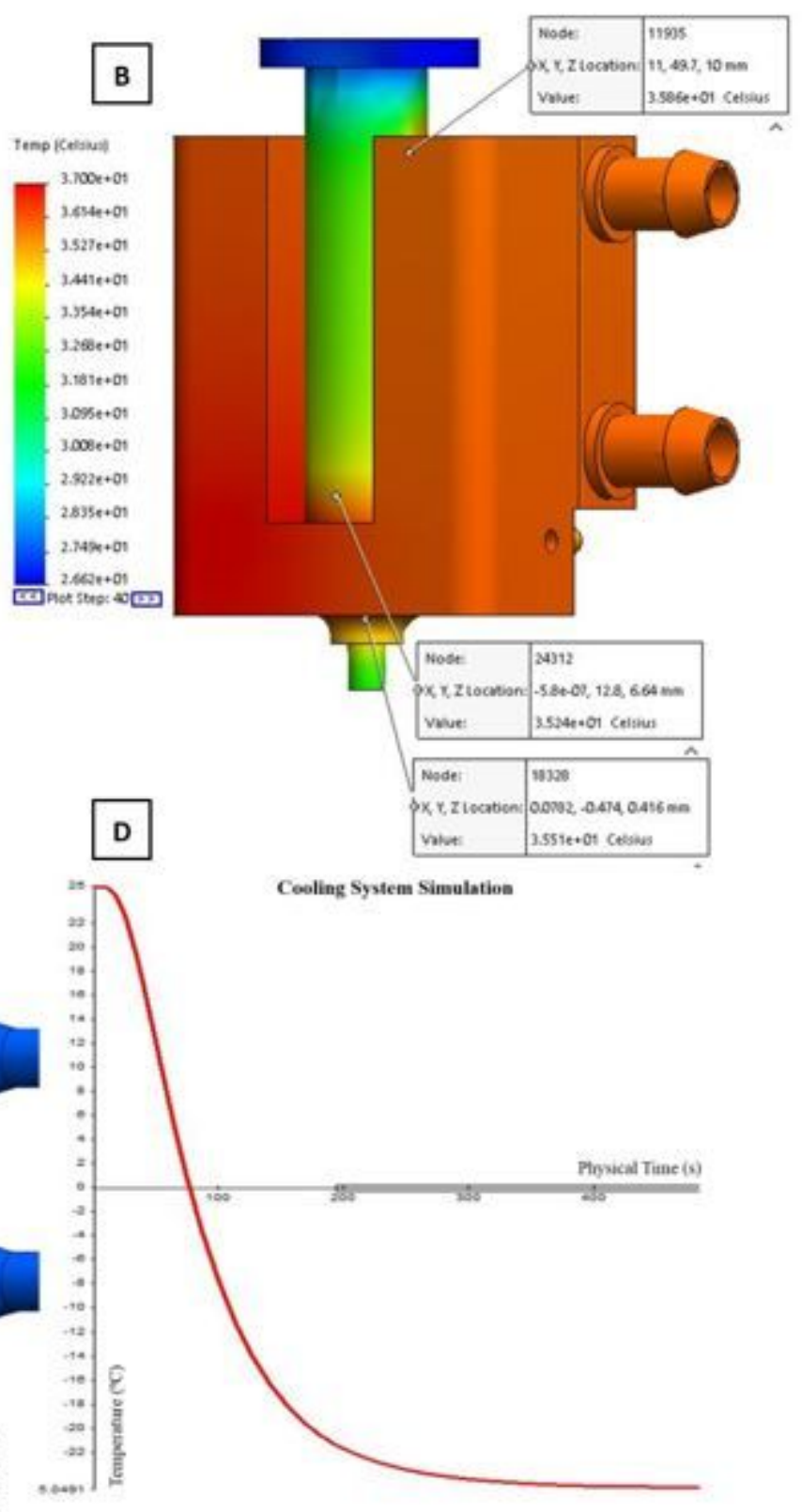

\section{Figure 6}

Heat transfer simulation plots for evaluation of thermal control system. (A) Temperature simulation plot in 400 seconds with heating target of $37^{\circ} \mathrm{C}$ in three different nodes of heating system. (B) 3-dimentional plot of the simulated system in the last step of simulation time series with heating target of $37^{\circ} \mathrm{C}$ in three different nodes of syringe cubic holder. (C) 3-dimentional plot of the simulated system in the last step of simulation time series with cooling target of $0^{\circ} \mathrm{C}$ in 78th second of simulation running time. (D) Temperature simulation plot in 400 seconds with cooling target of $0^{\circ} \mathrm{C}$ in the hydrogel loaded into the glass syringe which reached to a much higher performance as the curve was flatten in the region below the $-22^{\circ} \mathrm{C}$. 
A

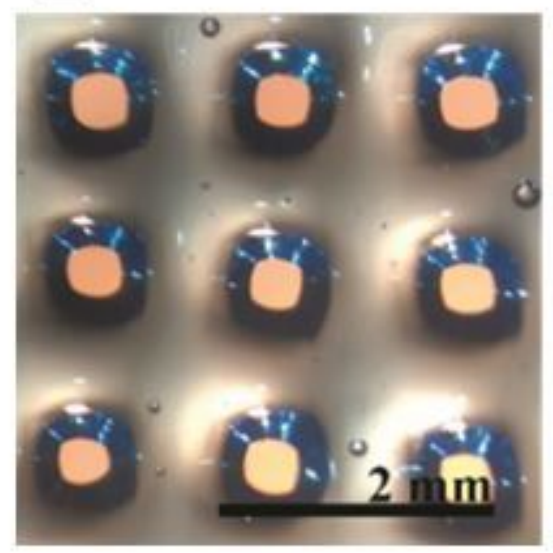

B

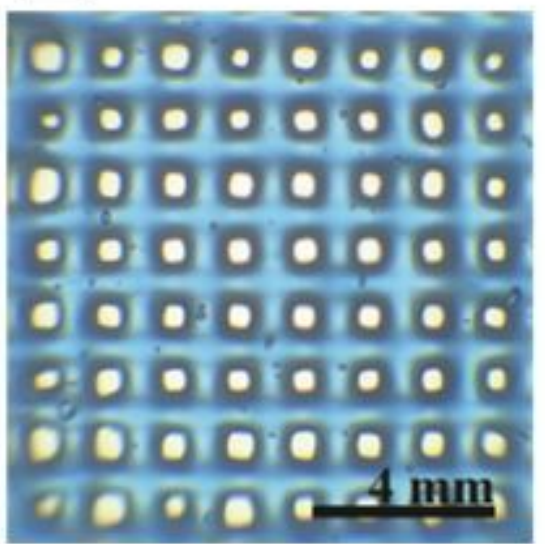

C

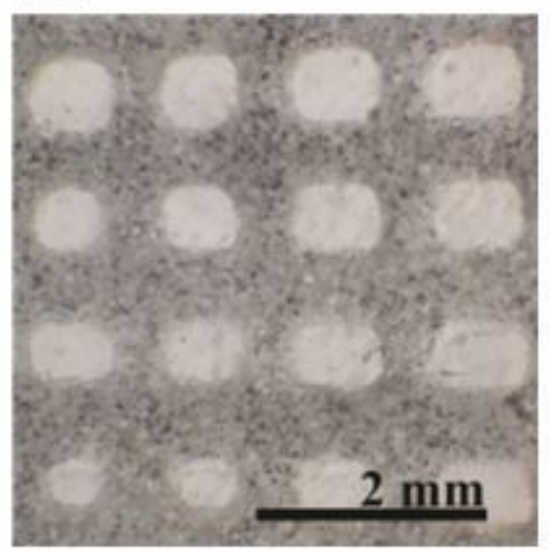

\section{Figure 7}

3D bio-printed hydrogel-based scaffold using portable multi-functional 3D bio-printing extrusion system with GelMa. (A) Stereo microscope image with 0.7 optical magnification; Scale bar $=2 \mathrm{~mm}$. (B) Inverted microscope image, $4 \mathrm{X}$ magnification; Scale bar $=4 \mathrm{~mm}$. (C) Inverted microscope image, $20 \mathrm{X}$ magnification; Scale bar $=2 \mathrm{~mm}$.

\section{Supplementary Files}

This is a list of supplementary files associated with this preprint. Click to download.

- Supplementaryinfo.docx

- SupplementaryVideoS1.avi 